
\title{
25 Research Soure \\ Berberine inhibits the proliferation of pancreatic cancer targeting pancreatic cancer stem cells
}

\section{Mengmeng Liu}

Dalian University of Technology https://orcid.org/0000-0003-0783-0990

Yue Pan ( $\triangle$ panyue0811@dlut.edu.cn )

Dalian University of Technology

\section{Xufeng Tao}

Dalian University of Technology

\section{Ning Li}

Dalian University of Technology

\section{Kun Li}

Dalian University of Technology

\section{Gary Guishan Xiao}

Dalian University of Technology

\section{Research}

Keywords: Berberine, PDAC, PCSCs, EMT, berberine

Posted Date: March 6th, 2021

DOI: https://doi.org/10.21203/rs.3.rs-282783/v1

License: (c) (i) This work is licensed under a Creative Commons Attribution 4.0 International License. Read Full License 


\section{Abstract}

\section{Background}

PDAC is universally acknowledged to be one of the highest mortality rate of cancer-related deaths. PCSCs, regulated by EMT, could promote the proliferation of PDAC. Berberine with high medicinal value has usually been used as an anti-cancer agent. Hence the purpose of this study is to investigate the anticancer effect of berberine in PDAC.

\section{Methods}

MTT assay was used to verify berberine inhibiting the proliferation of PDAC. Immunofluorescence staining, stem cell sphere, wound healing and transwell migration assay were demonstrated the antiproliferation and anti-stemness of PCSCs in vitro . PANC-02 cells were injected in C57BL/ 6 mice to establish the orthotopic pancreatic-cancer model in vivo. H\&E and Ki67 immunohistogical staining assay were used to evaluated the effect of berberine in PDAC in vivo. q-PCR and Western blot methods were applied to detect the expression of EMT procedure.

Results

In this study, berberine has selective anti-cancer effect in PDAC in vitro . Moreover, berberine suppressed the proliferation and stemness of PCSCs in PDAC. In vivo, berberine reduced the tumor size and decreased the expression of Ki67 in orthotopic pancreatic-cancer pancreases. In addition, berberine inhibit the EMT signaling pathway both in vitro and in vivo .

\section{Conclusions}

Our study indicates that berberine inhibit the proliferation of PDAC in vivo and vitro. The mechanism of anti-cancer effect on berberine may suppress the PCSCs through inhibiting EMT procedure. Therefore, berberine may be the novel antineoplastic drug with clinical effectiveness in PDAC. Keywords: Berberine, PDAC, PCSCs, EMT, berberine

\section{Full Text}

Due to technical limitations, full-text HTML conversion of this manuscript could not be completed. However, the manuscript can be downloaded and accessed as a PDF.

\section{Figures}


A

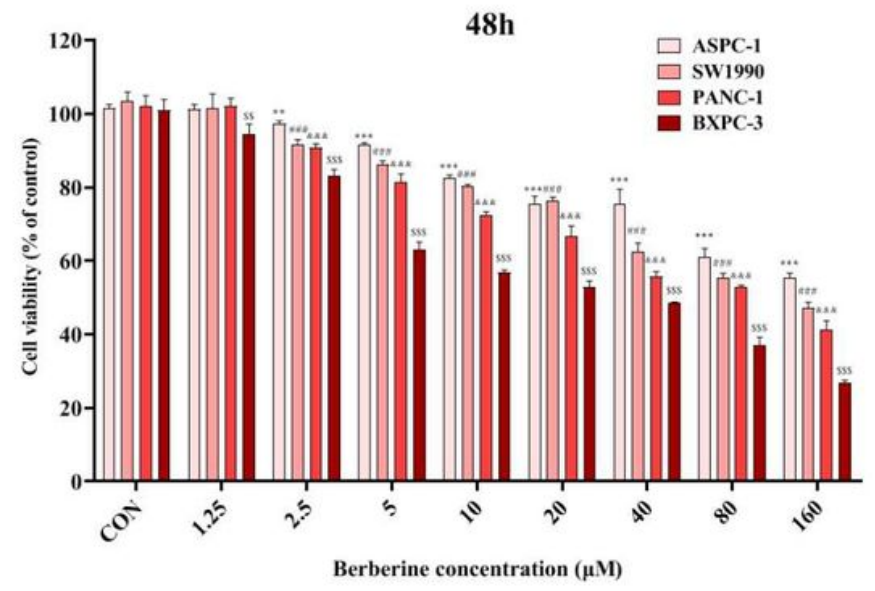

$\mathrm{C}$

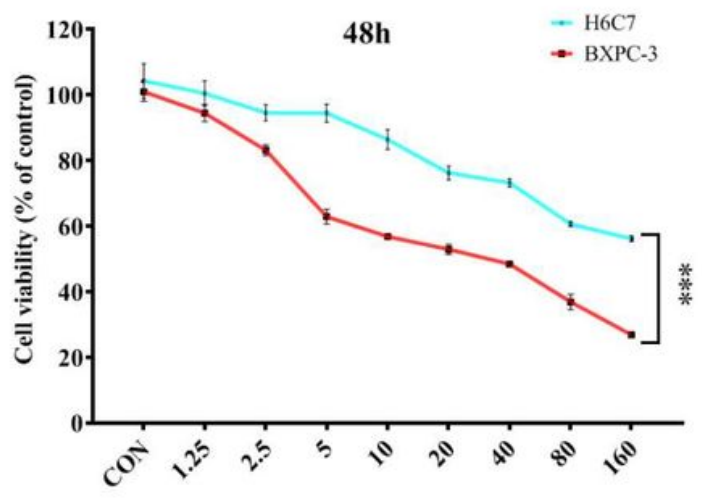

Berberine concentration $(\mu \mathrm{M})$
B

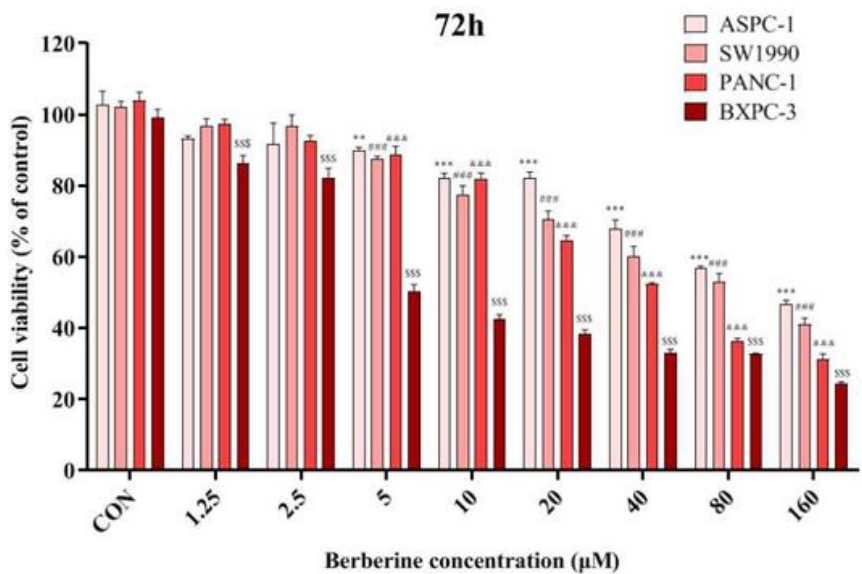

$\mathrm{D}$

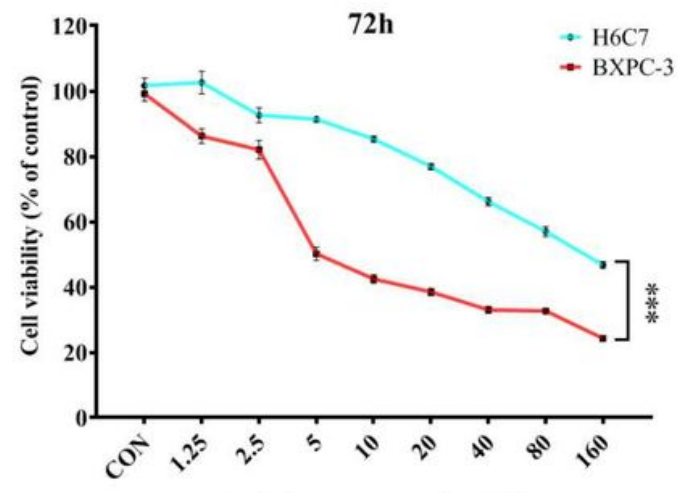

Berberine concentration $(\mu \mathrm{M})$

Figure 1

Effect of berberine on PDAC and normal pancreatic cell. (A and B) Cell viability treated with berberine in PANC-1, ASPC-1, SW1990, and BXPC-3. (C and D) Cell viability treated with berberine in BXPC-3 and H6C7. Data are expressed as mean \pm SD. ${ }^{*} P<0.05, \# P<0.05$, \&P<0.05, $\$ P<0.05$ versus $C O N, \star \star \star P<0.001$ versus $\mathrm{H} 6 \mathrm{C} 7$.

A
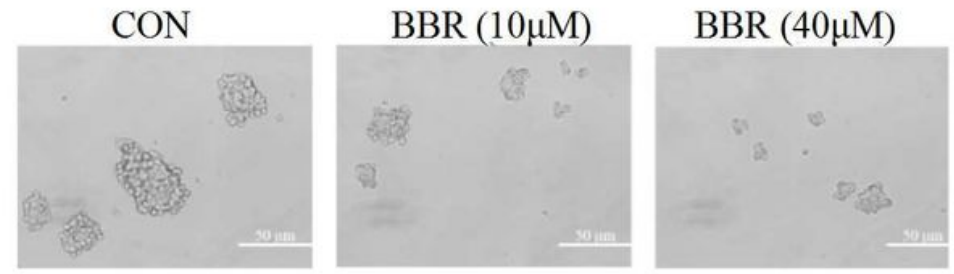

B

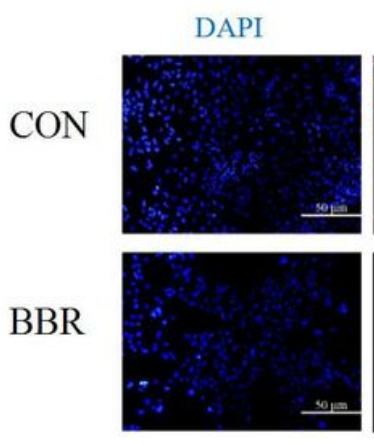

CD133

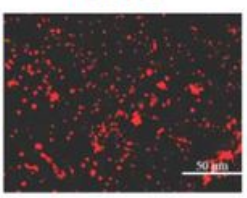

CD44
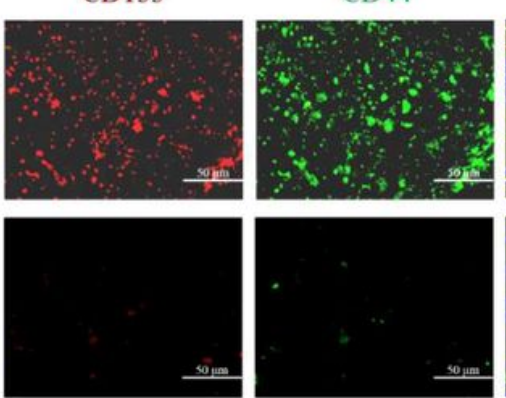

C
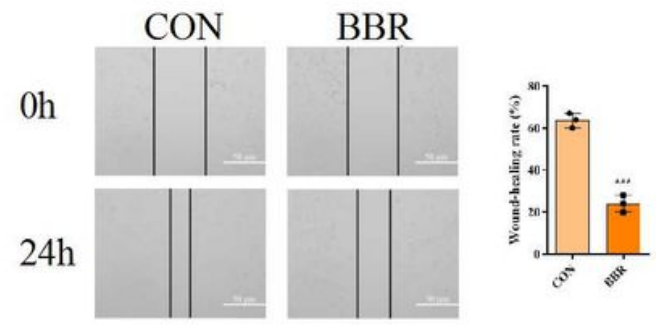

D

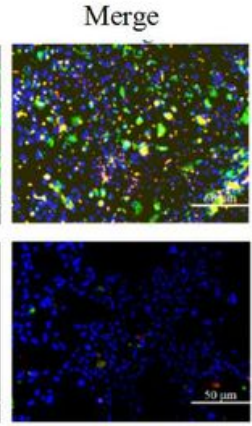

$\mathrm{CON}$

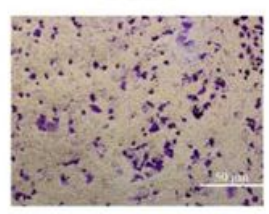

BBR

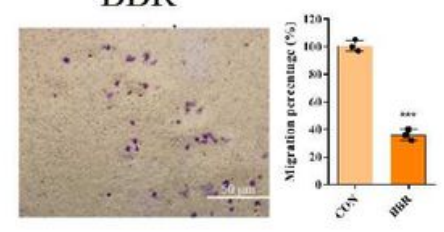


Figure 2

Effect of berberine on PCSCs stemness. (A) Sphere formation ability of berberine in PANC-1 cell. (B) Immunofluorescence of CD44 and CD133 in PANC-1 cell transfected with berberine for 24h. (C) Migration of berberine by wound healing assay. (C-D) Invasion of berberine by transwell model. Data are expressed as mean $\pm S D$. *** $\mathrm{P}<0.001$ versus $\mathrm{CON}$.

A

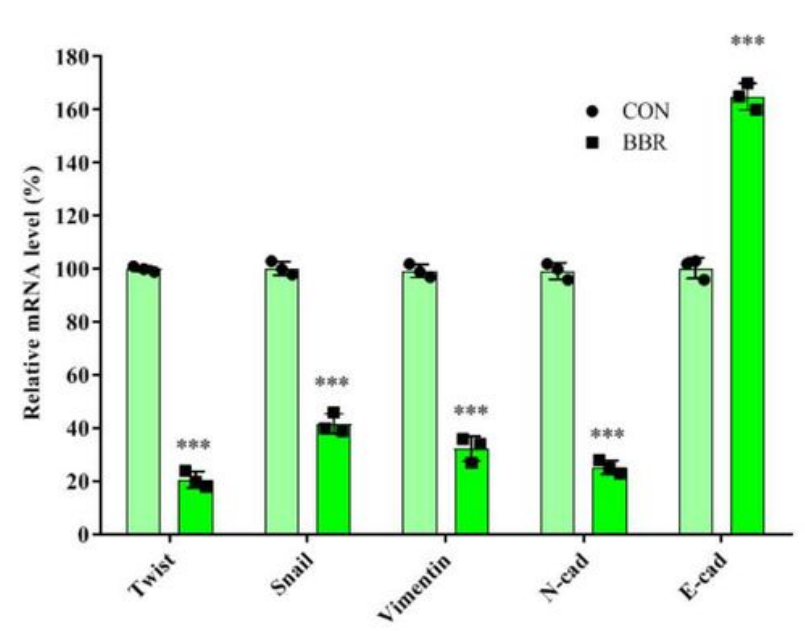

B

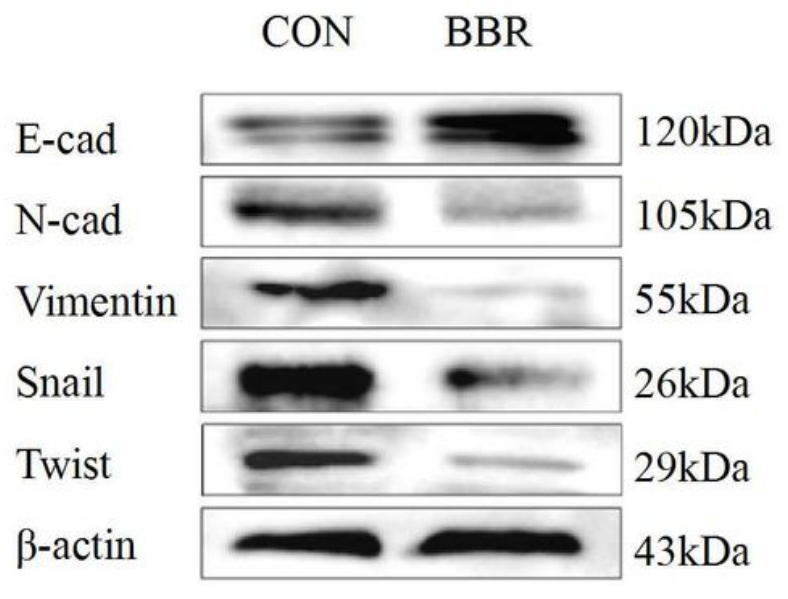

Figure 3

The effect of berberine on the EMT procedure in PANC-1 cell. (A) EMT relevant markers in mRNA level treated with BBR in PANC-1 cell. (B) EMT relevant markers in protein level treated with BBR in PANC-1 cell. Data are expressed as mean $\pm S D$. $* * * ~ P<0.001$ versus $C O N$.

A

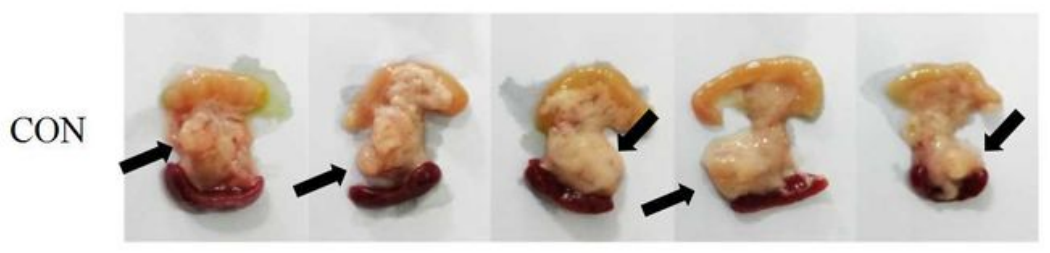

BBR

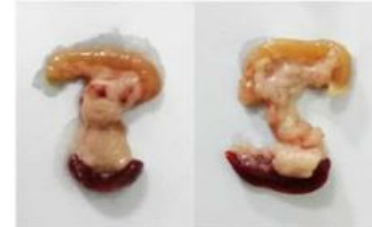

$\mathrm{C}$

CON

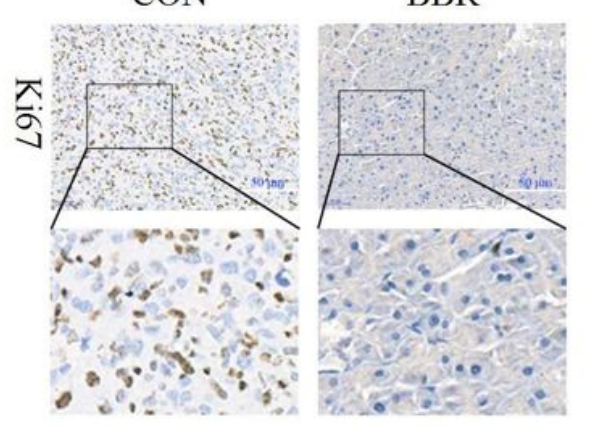

B

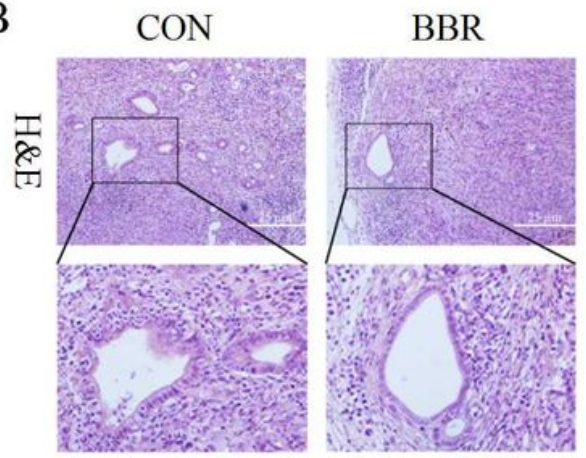

E

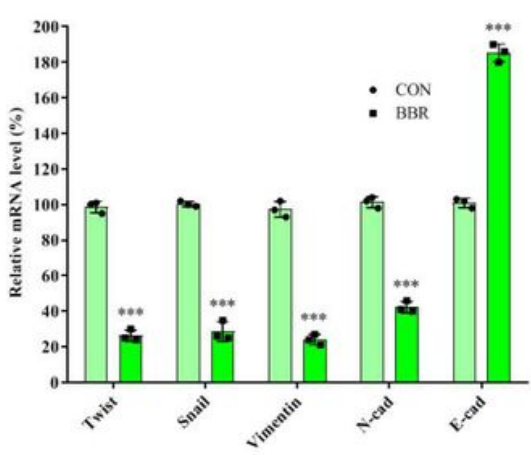

D

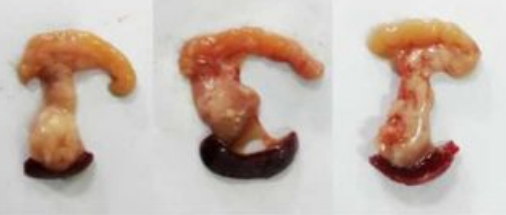

Page $4 / 5$ 
Figure 4

Effect of berberine in PDAC in vivo. (A) tumor photographs (B) H\&E staining in CON and BBR. (C) Ki67 $\mathrm{IHC}$ staining in CON and BBR. (D) EMT relevant markers in mRNA level treated with BBR in pancreatic tissue. (E) EMT relevant markers in protein level treated with BBR in pancreatic tissue. Data are expressed as mean $\pm S D$. ${ }^{\star * \star} P<0.001$ versus $C O N$.

\section{Supplementary Files}

This is a list of supplementary files associated with this preprint. Click to download.

- SupplementaryFigure1.jpg 